\title{
Role of caloric vestibular stimulation in improvement of motor symptoms and inhibition of neuronal degeneration in rotenone model of Parkinson's disease - An experimental study
}

\author{
J. THANALAKSHMI ${ }^{1}$, R. ARCHANA ${ }^{1 *}$ (1) S. SENTHILKUMAR ${ }^{2}$, \\ R. SHAKILA ${ }^{3}$, N. PAZHANIVEL ${ }^{4}$ and S. SUBHASHINI ${ }^{3}$
}

\footnotetext{
${ }^{1}$ Department of Physiology, Saveetha Medical College Hospital, Saveetha Nagar, Thandalam, Chennai 602105, Tamil Nadu, India

${ }^{2}$ Department of Research and Development, Saveetha Institute of Medical and Technical Sciences, Chennai 602105, Tamil Nadu, India

${ }^{3}$ Department of Chemistry, Siddha Central Research Institute, Arumbakkam, Chennai, India

${ }^{4}$ Department of Veterinary Pathology, Madras Veterinary College, Chennai 602105, India
}

Received: March 28, 2020 • Accepted: July 27, 2020

Published online: October 3, 2020

(C) 2020 Akadémiai Kiadó, Budapest

\begin{abstract}
Objective: Parkinson's disease (PD) is a progressive neurodegenerative disorder. In order to explore a noninvasive treatment of $\mathrm{PD}$, in the current study the authors evaluated the neuroprotective efficacy of caloric vestibular stimulation (CVS) using the rotenone-induced rat model of PD. The rotenone models of $\mathrm{PD}$ are gaining attention due to high reproducibility. It is also considered to be an improved model to exhibit the pathogenesis of PD and test the neuroprotective effect of various therapeutic interventions. Materials and methods: Rotenone was i.p. injected ( $3 \mathrm{mg} / \mathrm{kg}$ body weight) to male Wistar albino rats for 21 days to induce PD. As PD is chronic and progressive in nature, the efficacy of chronic CVS intervention was evaluated for 30 days after inducing PD in rats. Motor symptoms were evaluated by assessing locomotor activity in actophotometer, whereas movement analysis was done using Ludolph test and motor coordination was evaluated using rotarod apparatus. The neurochemical and neuropathological changes were also observed in the corpus striatum of rats. Results: Rotenone administration showed decreased
\end{abstract}

\footnotetext{
* Corresponding author. Saveetha Medical College and Hospital, Saveetha Institute of Medical and Technical Sciences, Thandalam, Chennai - 602 105, India. Tel.: +91 9840608149. E-mail: dr.rarchana@gmail.com
} 
locomotor activity, motor coordination and general movement associated with significant $(P<0.05)$ reduction in dopamine content in the corpus striatum. The immunohistochemical analysis revealed a marked decrease in tyrosine hydroxylase $(\mathrm{TH})$ immunoreactivity in striatal neurons indicating the significant loss of dopaminergic neurons in substantia nigra (SN) following rotenone injection. However, chronic treatment with CVS restored the nerve terminals in the striatum from rotenone damage. CVS treatment improved the dopaminergic system function by restoring dopamine content in the striatum. CVS also improved the motor deformities clearly suggesting the neuroprotective function. Conclusion: The results of the present study suggested CVS to be a safe and simple neuroprotective measure against neurodegenerative changes in PD and a promising noninvasive technique to overcome the motor symptoms associated with it. The findings could be useful for further investigations and clinical applications of CVS in the treatment of PD.

\section{KEYWORDS}

parkinson's disease, rotenone, rotarod, actophotometer, dopamine, tyrosine hydroxylase, caloric vestibular stimulation

\section{INTRODUCTION}

Parkinson's disease (PD) is a progressive degenerative disorder of the dopaminergic neurons of substantia nigra $(\mathrm{SN})$. The cardinal biochemical abnormality in PD is the profound deficit in brain dopamine levels, primarily contributing to the loss of neurons in the nigrostriatal dopaminergic pathway. The pathway consists of dopaminergic neurons whose cell bodies are situated in the SN pars compacta and whose axons and nerve terminals project to the striatum [1]. PD is associated with motor deformities like bradykinesia, rigidity, and tremor contributing to decline in balance and mobility [2]. The nonmotor symptoms associated with PD are diverse like cognitive problems such as apathy, depression, anxiety disorders, and hallucinations, sleep disorders, sexual dysfunction, bowel problems and dribbling of saliva [3-5]. The most widely used animal models of PD involve intraperitoneal injection of rotenone in which the animals exhibit both motor and nonmotor deficits of the disease [6]. Several efforts were made to develop replacement therapeutic strategies with L-dopa and dopamine agonists to control the early and later stages of PD. Even though L-dopa treatment was found to be effective, when given for longer duration, the excessive activation of cerebral dopamine metabolism promoted oxidative stress. As a result, an increase in the rate of cell degeneration was observed in SN, which in turn potentiated PD progression in humans [7]. Although L-dopa is the standard therapy of care in PD, many dopamine-indepedent treatments have been available (e.g., deep brain stimulation (DBS)). Nevertheless, the effects of DBS were controversial in terms of stimulation-related psychiatric disturbances. Moreover, the inclusion criteria for DBS were restricted to significant motor disability with absence of cognitive dysfunction, which was not suitable for elderly patients [8]. Recent studies suggested that galvanic vestibular stimulation could improve cognition in PD [9]. However, some authors reported discomfort while applying the current and was not suitable for elderly patients [10].

Caloric vestibular stimulation (CVS) is a simple and noninvasive physiological measure that could relieve stress [11] and improve the motor and nonmotor symptoms of PD patients [12, 
13]. However, the underlying molecular mechanisms behind the benefits of CVS remain unclear and less explored. The current study was conducted with the objective to examine the effects of bilateral CVS on behavioral parameters, changes in dopamine levels and histopathological changes in the striatum of rats with rotenone-induced PD.

\section{MATERIAL AND METHODS}

\section{Drugs and chemicals}

Rotenone, dimethylsulfoxide (DMSO), reference standard dopamine and analytical grade reagents were procured from Sigma-Aldrich, USA. Anti-TH mouse monoclonal primary antibody and anti-mouse IgG secondary antibody were purchased from Thermo Fisher Scientific, USA.

\section{Experimental animals}

The study was carried out after having received ethical clearance from the Institutional Animal Ethics Committee (Approval number - SU/CLAR/RD/006/2018). Efforts were made to use a minimal number of animals for the study and to reduce animal suffering. The subjects $(n=24)$ were male Wistar albino rats, weighing approximately $200-225 \mathrm{~g}$. The animals were housed in a room kept at appropriate room temperature and equipped with light control. The animals were fed and allowed to drink water ad libitum. Rats were treated in accordance with the guidelines of CPCSEA. The behavioral experiments were carried out between 9.00 and $12.00 \mathrm{~h}$.

\section{Rotenone administration}

In the present study, PD was induced by intraperitoneal injection of rotenone at a dose of $3 \mathrm{mg} /$ $\mathrm{kg}$ body weight/day for 21 days. A rotenone stock solution was first prepared in DMSO and diluted with olive oil to obtain a final concentration of $3 \mathrm{mg} / 5 \mathrm{~mL}$ rotenone. The solution was made fresh and stored in an amber bottle protected from light. The solution was vortexed before administration to eliminate the possibility of settling. The procedure used in the current study for inducing PD in rats was adopted from the previous work with slight modification [2]. The control group received only vehicle (99\% olive oil and $1 \%$ DMSO) of $1 \mathrm{~mL} / \mathrm{kg}$ body weight.

The mortality rate during the experiment was maintained at a minimum by careful needle placement during injection, administration of appropriate doses of rotenone and mixing the solution using a vortexer before each i.p. injection. During the study, one rat from the PD group died (on second day).

\section{Caloric vestibular stimulation}

The middle ear cavity of each ear was irrigated using a polyethylene tube with $2 \mathrm{~mL}$ of warm water at $42{ }^{\circ} \mathrm{C}$ to study the efficacy of CVS. The flow rate was maintained at $0.1 \mathrm{~mL} / \mathrm{s}$ to induce constant vestibular stimulation $[14,15]$. The rats were held in position such that the horizontal canal was tilted approximately $30^{\circ}$ with respect to the horizontal plane to produce convection currents in the semicircular canals. 


\section{Experimental design}

The experimental rats were randomly assigned into four different groups (each group having 6 animals).

Group I: Control group administered with olive oil as vehicle (CONT).

Group II: PD group i.p. injected with rotenone (PD).

Group III: Rotenone i.p. injection and CVS for 30 days (PD + CVS).

Group IV: Only CVS for 30 days (CVS).

Rats in the PD + CVS group were injected with rotenone for 21 days to induce PD (confirmed by behavioral changes), following which CVS was administered for 30 days. The body weights of all the study animals were recorded prior to the start of experiments and behavioral assessment was done at various time points, as mentioned below.

\section{Time line for behavioral assessment}

T1: Behavioral testing was performed 1 day prior to the start of rotenone injections (Pre injection Day 1).

T2: Recording was done after continuous rotenone injection for 10 days (injection period Day 10).

T3: Further treatment of rotenone for 11 days and behavioral testing (injection period Day 21).

T4: Behavioral recording after 30 days of CVS administration without any further rotenone injection (Post injection period Day 30).

After the interventional duration of 30 days, all the animals were decapitated, their brains removed and immediately processed for high performance liquid chromatography (HPLC) and histopathological analysis.

\section{BEHAVIORAL ASSESSMENT}

\section{General movement analysis}

The rotenone-induced motor deformity was assessed using a neurological scale by Ludolph et al. [16]. A general behavioral score was set for each animal prior to the start of experiment, and then regularly at 10-day intervals. The scoring scheme is as follows: score $=0$ : normal behavior, 1: general slowness of displacement due to mild hind limb impairment, 2: in-coordination and marked gait abnormalities, 3 : hind limb paralysis, 4 : incapacity to move resulting from forelimb and hind limb paralysis, and 5: recumbency.

\section{Actophotometer}

The spontaneous locomotor activity of rats was studied using the actophotometer (Inco, India). It was recorded by placing the animal in the center square arena and the animal ambulatory activity was noted continuously for $5 \mathrm{~min}$. When the beam of light falling on the photocell was cut off by the animal movement, the digital data displayed as a count on the front panel meter was recorded [1]. 


\section{Rotarod test}

The accelerating digital rotarod apparatus (INCO Instruments \& Chemicals Pvt. Ltd, India) was used to assess the motor coordination of the rats. The apparatus consists of a rotating horizontal metal rod coated with rubber $(3 \mathrm{~cm}$ diameter). All rats were trained for 5 days prior to the start of the experiment. The rotation speed was fixed at $12 \mathrm{rpm}$ on the first day of training and reached $15 \mathrm{rpm}$ on the last day of training. The cut-off time was fixed as $180 \mathrm{~s}$ and the rat was made to perform three test trials, with 5 min gap in between. A test trial was stopped when the rat fell off the rotating rod or completed the cut-off time of $180 \mathrm{~s}$. The mean value of three trials was taken as a reading [17].

\section{DETERMINATION OF DOPAMINE IN STRIATUM BY HIGH PERFORMANCE LIQUID CHROMATOGRAPHY}

\section{Sample preparation}

The experimental rats were sacrificed by cervical dislocation and the corpus striatum was dissected and separated out quickly on an ice-cold plate, weighed, and preserved in liquid nitrogen. The tissue was homogenized with perchloric acid and centrifuged at $12,000 g, 4{ }^{\circ} \mathrm{C}$ for 2 min and the supernatant was collected for determination of dopamine in the corpus striatum. The collected supernatant was again centrifuged at $12,000 \mathrm{~g}, 4^{\circ} \mathrm{C}$ for $20 \mathrm{~min}$ and filtered through a $0.22 \mu \mathrm{m}$ membrane filter. The collected filtrate $(1 \mathrm{~mL})$ was added into a sterile dark container and stored at $-80{ }^{\circ} \mathrm{C}[18]$.

\section{Estimation of dopamine}

Dopamine contents were estimated by LC-20AP HPLC (Shimadzu Corporation, Kyoto, Japan) with photodiode array detector (PDA) detector as adopted by $\mathrm{Gu}$ et al. [18]. The system was equipped with degassing unit, column oven, low pressure gradient and isocratic unit, LC-20AP Quaternary low pressure mixing pump and Shimadzu SPD-M20A UV-DAD detector with thermostated flow cell. Also, a shim pack RP-C ${ }_{18}$ column $(150 \times 4.6 \mathrm{~mm} ; 3 \mu)$ with $20 \mu \mathrm{L}$ aliquots sample loop volume and $20 \mu \mathrm{L}$ Hamilton injector was used. The data were collected and analysis was done in the HPLC system using Lab Solution software. The mobile phase was $5 \mathrm{mM}$ $\mathrm{HClO}_{4}$ solution containing $5 \%$ acetonitrile, the flow rate was 0.2 or $0.25 \mathrm{~mL} / \mathrm{min}$ and the separation temperature was $30{ }^{\circ} \mathrm{C}$. The selection of the suitable wavelength for dopamine detection was important, and therefore optimal detection range was set at $277 \mathrm{~nm}$. Stock solutions $(10 \mu \mathrm{g} / \mathrm{mL})$ of dopamine were prepared by dissolving the standard in $0.2 \mathrm{M} \mathrm{HClO}_{4}$ solution. The solution was refrigerated and stored in a dark container. The samples $(20 \mu \mathrm{L})$ and standards at various concentrations were prepared and injected manually.

\section{Histopathological evaluation of corpus striatum by hematoxylin and eosin (H\&E) staining}

The animals were anesthetized and perfused intracardially with physiological saline as prerinse followed by perfusion with fixative solution of neutral buffered formalin (10\%). The brain section containing corpus striatum (approximately $0.20 \mathrm{~mm}$ relative to bregma) was removed 
and postfixed with neutral buffered formalin (10\%) for about 24-48 h. After fixation, tissues were dehydrated in ascending grades of alcohol, embedded in paraffin wax, following the standard technique. Paraffin sections of 5-7 $\mu \mathrm{m}$ thickness were cut using microtome and then subjected to hematoxylin \& eosin staining (Bancroft and Gamble 2008). The histopathological assessment of the corpus striatum of brain tissue was performed under a BX51 Olympus multi head microscope [19].

\section{Immunohistochemical assessment of tyrosine hydroxylase immunoreactivity}

The sections of corpus striatum (approximately $0.20 \mathrm{~mm}$ relative to bregma) were dissected using a microtome, rinsed in Phosphate buffered saline (PBS) and incubated in $1 \% \mathrm{H}_{2} \mathrm{O}_{2}$ for 30 min to block the endogenous peroxidase activity. After washing in PBS, the sections were incubated in blocking serum (10\% normal horse serum and $0.1 \%$ Triton X-100 in PBS) for 60 min, followed by incubation in anti-TH mouse monoclonal antibody solution (1:500) for $24 \mathrm{~h}$ at room temperature. The sections were then incubated for $1 \mathrm{~h}$ in biotinylated anti-mouse IgG secondary antibody (1:300). The sections were subsequently incubated with avidin-biotinperoxidase complex for $1 \mathrm{~h}$ at room temperature. Immunoreactivity was visualized by incubating the sections in a solution consisting of $0.05 \%$ 3,3-diaminobenzidine (DAB) and $0.02 \%$ $\mathrm{H}_{2} \mathrm{O}_{2}$ in $50 \mathrm{mM}$ Tris buffer ( $\mathrm{pH} \mathrm{7.6)}$ for $3 \mathrm{~min}[2]$.

\section{Statistical analysis}

Descriptive data were expressed as mean \pm standard error of the mean (SEM). For all the tests, statistical significance was set as $P<0.05$. The data for behavioral studies were analyzed using three-way ANOVA with repeated measures (Dunnett T3 multiple comparison test between various combinations). The data for dopamine estimation were analyzed using one-way ANOVA followed by Student-Newman-Keuls test.

\section{RESULTS}

\section{CVS prevents rotenone-induced behavioral changes}

In the present study, PD was induced in rats by i.p. administration of rotenone, as it caused motor deformities. After rotenone injection, the behavioral activities such as locomotion, general movement and motor coordination were significantly reduced in rats, which was later observed to improve, following the CVS administration.

\section{Body weight}

Analysis using three-way ANOVA with weeks as within-animals factor was found to be significant $(P<0.05)$. Dunnett T3 multiple comparison test was carried out to test the difference between the groups. There was a significant $(P<0.05)$ decrease in body weight between the rotenone (PD) and the control groups. Body weight increased after CVS, which was not statistically significant. However, there was no difference between the control and CVS groups (Fig. 1). 


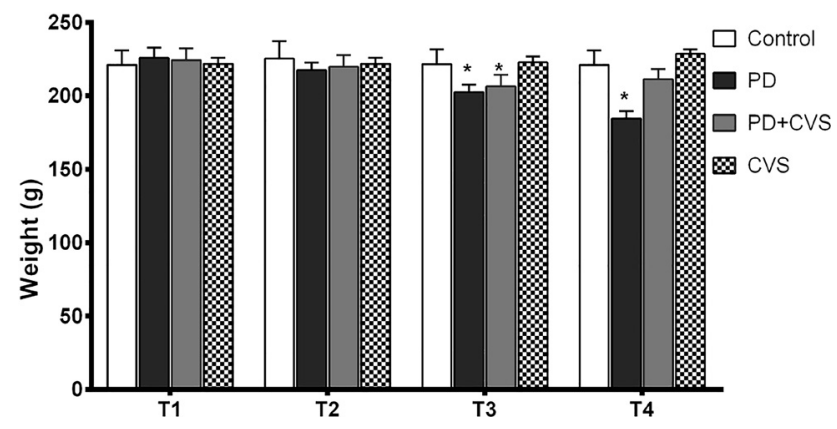

Fig. 1. Effect of caloric vestibular stimulation on body weight of rats with rotenone-induced PD at various time points (T1: Pre injection day 1; T2: Injection period day 10; T3: Injection period day 21; T4: Post injection period day 30). Data are expressed as mean score \pm SEM and ${ }^{*}(P<0.05)$ was considered to be statistically significant as compared to the control group

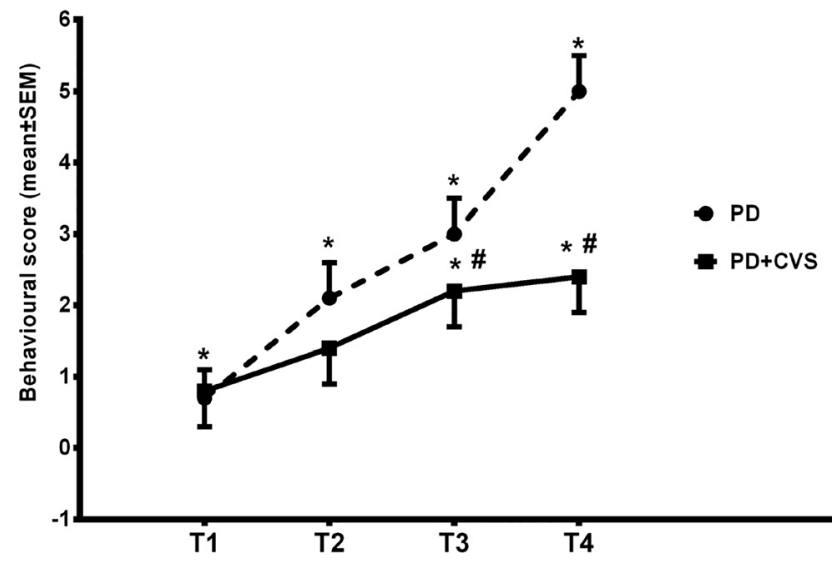

Fig. 2. Effect of caloric vestibular stimulation on Ludolph scale movement analysis of rats with rotenoneinduced PD at various time points (T1: Pre injection day 1; T2: Injection period day 10; T3: Injection period day 21; T4: Post injection period day 30$)$. Data are expressed as mean score \pm SEM. ${ }^{*}(P<0.05)$ was considered to be statistically significant as compared to the control group and ${ }^{\#}(P<0.05)$ was significant as compared to the PD group

\section{Movement analysis by Ludolph scale}

Analysis using three-way ANOVA with weeks as within-animals factor was found to be significant for movement analysis $(P<0.05)$. Also, the three-way interaction between weeks and groups was found to be significant $(P<0.05)$. Three-way ANOVA with various groups as between-subjects factor was also found to be significant for movement analysis $(P<0.01)$. Multiple 


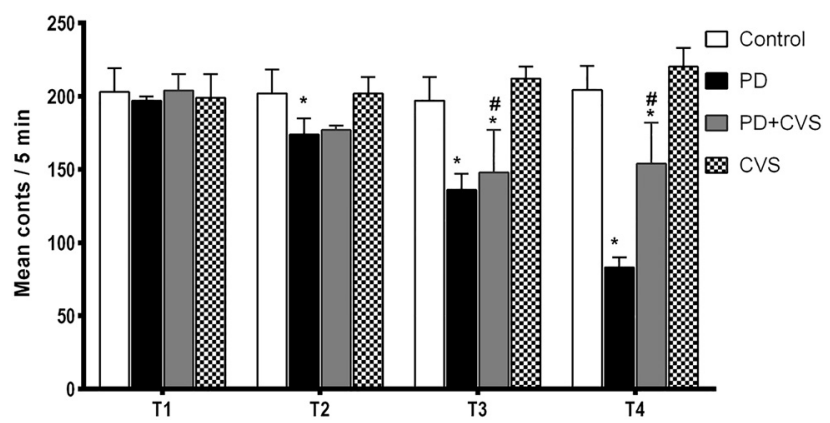

Fig. 3. Effect of caloric vestibular stimulation on locomotor activity assessed by actophotometer at various time points (T1: Pre injection day 1; T2: Injection period day 10; T3: Injection period day 21; T4: Post injection period day 30$)$. Data are expressed as mean activity $\pm \operatorname{SEM}^{*}{ }^{*}(P<0.05)$ was significant as compared to the control group and ${ }^{\#}(P<0.05)$ was significant as compared to the PD group

comparisons with Dunnett T3 test were carried out, and it was found that there was a significant reduction in motor activity in the PD group when compared to the control group $(P<0.05)$. Movement analysis showed a significant difference between PD and PD + CVS groups $(P<$ 0.01). No difference was found between the control and the CVS groups for movement analysis, as the rats exhibited normal behavior with zero score (Fig. 2: Data for control and CVS not represented in graph).

\section{Locomotor activity assessment by actophotometer}

Analysis using three-way ANOVA with weeks as within-animals factor was found to be significant for locomotor activity analysis $(P<0.05)$. Also, the three-way interaction between weeks and groups was found to be significant $(P<0.05)$. Three-way ANOVA analysis with various groups as between-subjects factor was also found to be significant for locomotor activity $(P<$ 0.051). Multiple comparisons between the groups showed a significant decrease in total locomotor activity in the rotenone group (PD) when compared to the control group. The locomotor activity showed a significant difference between the PD and PD + CVS groups $(P<0.05)$. There was no significant difference observed between the control and CVS groups for movement analysis (Fig. 3).

\section{Motor coordination assessment by rotarod test}

Three-way ANOVA analysis with weeks as within-animals factor was found to be significant for the rotarod test $(P<0.05)$. Furthermore, the three-way interaction between weeks and groups was found to be significant $(P<0.05)$. Three-way ANOVA analysis with various groups as between-subjects factor was also found to be significant for motor coordination $(P<0.051)$. The results of multiple comparisons between the groups showed a significant decrease in time taken for the animal to fall $(P<0.01)$ in $\mathrm{PD}$-induced rats when compared to rats of the control group. There was a significant increase in fall-off time $(P<0.001)$ after intervention with CVS for 30 


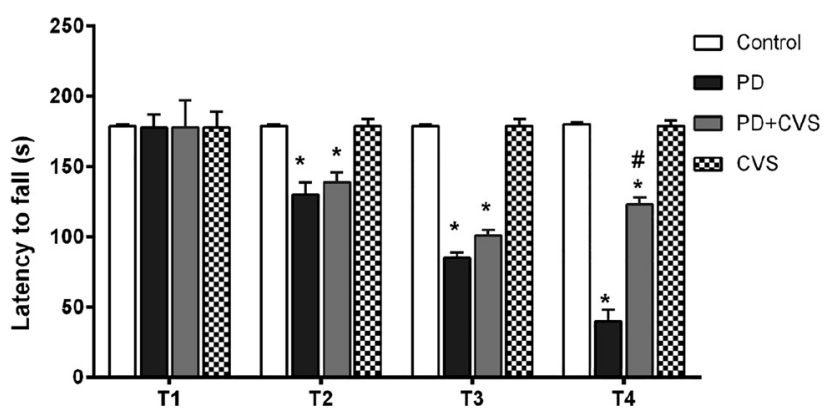

Fig. 4. Effect of caloric vestibular stimulation on rotarod performance at various time points (T1: Pre injection day 1; T2: Injection period day 10; T3: Injection period day 21; T4: Post injection period day 30). Data are expressed as mean value \pm SEM. ${ }^{*}(P<0.05)$ was significant as compared to the control group and ${ }^{\#}(P<0.05)$ was significant as compared to the PD group

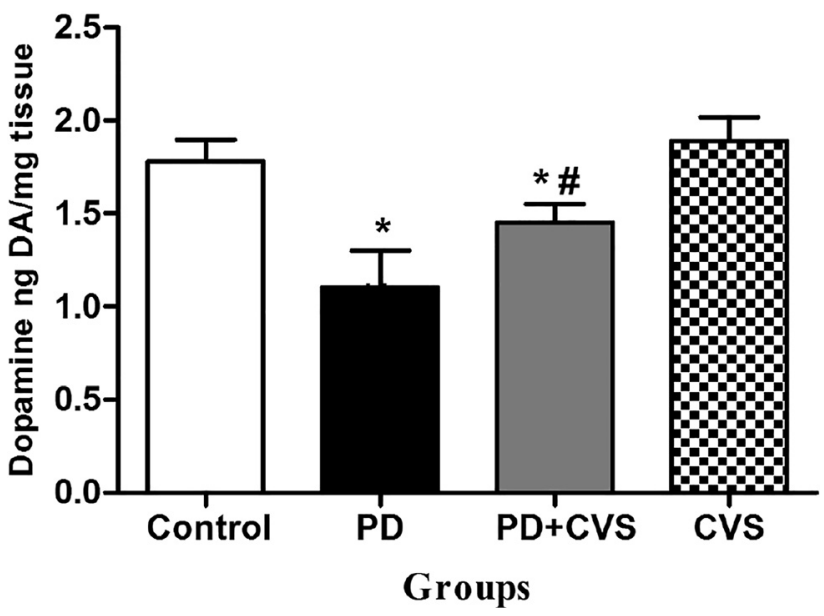

Fig. 5. Effect of caloric vestibular stimulation on concentration of dopamine (ng) in corpus striatum of control rats, PD rats, $\mathrm{PD}+\mathrm{CVS}$ rats and CVS rats. Values are expressed as average \pm SEM. ${ }^{*}(P<0.05)$ was significant as compared to the control group and ${ }^{\#}(P<0.05)$ was significant as compared to the PD group

days $(P<0.001)$, when compared to the PD group. The authors did not observe a significant difference between the CVS only group and the control group (Fig. 4).

\section{CVS inhibited rotenone-induced dopamine dysfunction in the corpus striatum}

Based on the beneficial effect revealed by CVS against rotenone-induced motor impairment, the dopamine concentration in the corpus striatum was measured. The statistical analysis showed that there was a significant decrease in dopamine level, indicating dysfunction in the PD group 

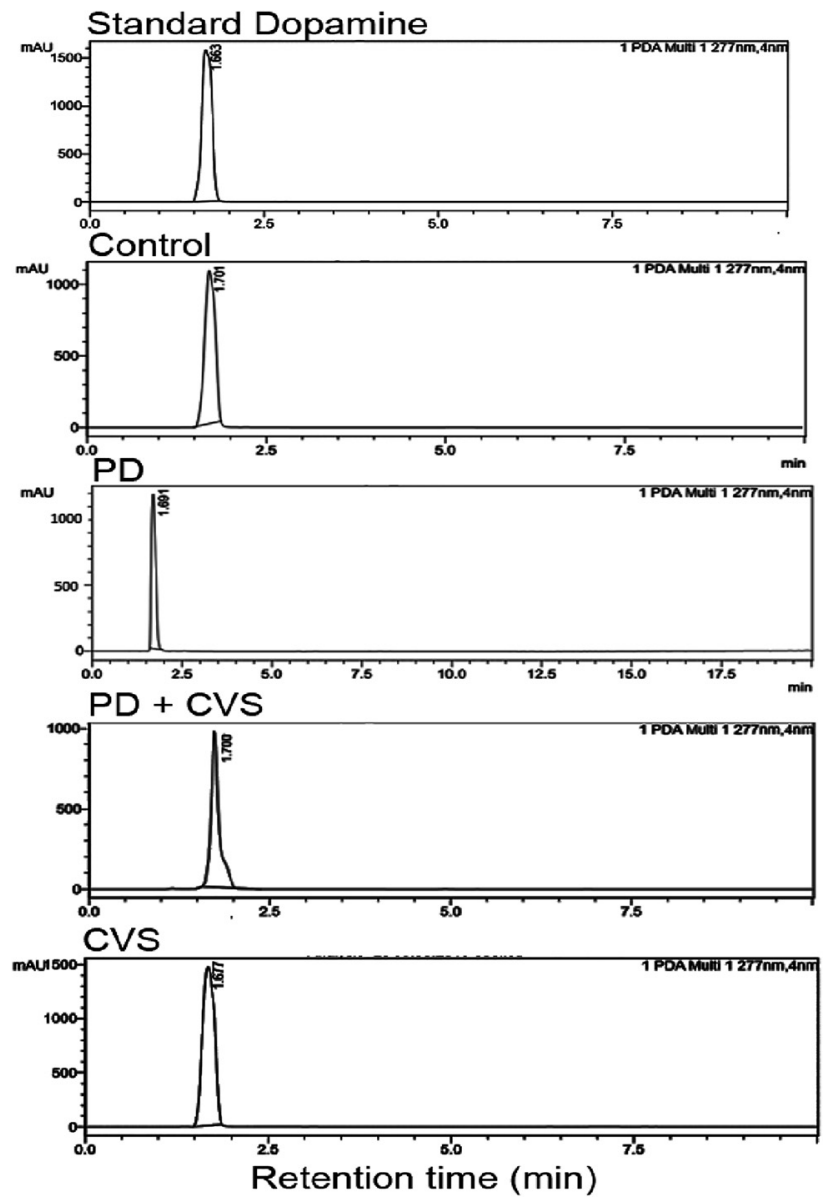

Fig. 6. HPLC chromatogram showing dopamine peak (DA) in rat brain tissue homogenates of control group, PD group, PD + CVS group and CVS group

when compared to the control group $(P<0.05)$. There was a significant improvement in dopamine concentration after CVS administration in the PD + CVS group indicating the protective function. There was no difference between the control and CVS groups, suggesting that there was no adverse effect caused by CVS (Figs. 5 and 6).

\section{CVS reduced loss of dopamine-secreting neurons in the corpus striatum of rotenone- treated animals}

In the present study, the neuroprotective function of CVS was studied through the histopathological changes in the corpus striatum. The control group showed normal pyramidal cells with nuclei and intact cell membranes. Following the rotenone injection, there was a significant 

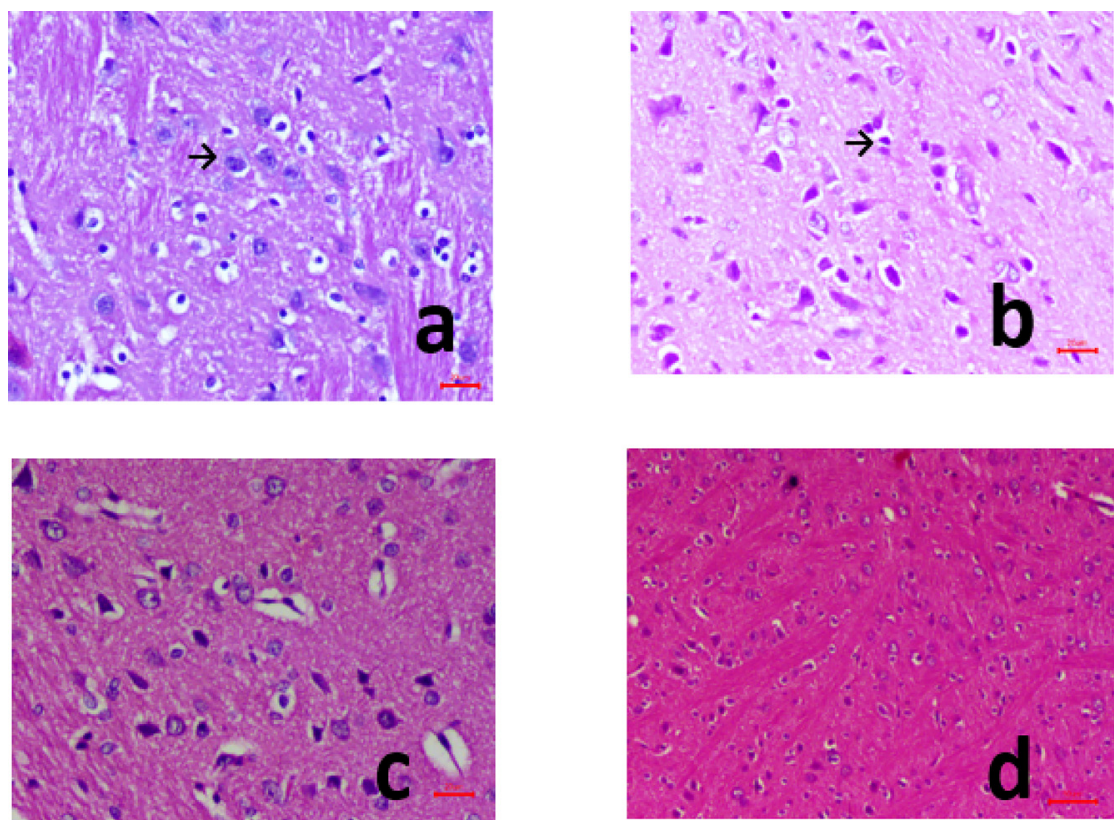

Fig. 7. Histopathological microscopic images of corpus striatum stained with hematoxylin and eosin (H\&E). (A) control, arrow head indicating pyramidal neurons with blue cytoplasm and distinct nucleolus (B) PD, arrow head indicating neurons with eosinophilic, shrunken and pyknotic nuclei (C) PD+CVS, showing reduced number of eosinophilic shrunken and pyknotic nuclei in the neurons indicating reduction in degenerated neurons (D) CVS, evident with vital neurons with clear nucleus (scale bar $20 \mu \mathrm{m}$ )

decrease in the number of normal neurons and severe to moderate spongiosis was also observed in the striatum. Multifocal neurons revealed degeneration characterized by the appearance of eosinophilic, shrunken, and pyknotic nuclei. Few apoptotic neurons were also seen with reactive astrocytes. Furthermore, the intervention with CVS for 30 days resulted in a reduced number of eosinophilic, shrunken and pyknotic nuclei in the neurons, indicating recovery of neurons from the degeneration. In the corpus striatum of the CVS group, the vital medium-sized spiny neurons with clear nuclei and distinct nucleoli in the center were evident (Fig. 7).

\section{TH immunoreactivity}

The dopaminergic neurons in SN project their processes to the striatum where the terminal fibers will be enriched with dopamine and its receptors. The dopamine concentration and the neuronal changes in the striatum were assessed and the associated dysfunction was noted after rotenone injection. Therefore, the authors examined the loss of dopaminergic neurons in the corpus striatum by evaluating the intensity of tyrosine hydroxylase $(\mathrm{TH})$ immunoreactivity. $\mathrm{TH}$ is the rate-limiting enzyme for the synthesis of dopamine, and its expression reflects the dopamine level in the catecholaminergic neurons. The results of TH expression (Fig. 8) in the neurons of corpus striatum was less in PD rats, indicating degeneration and damage to the 

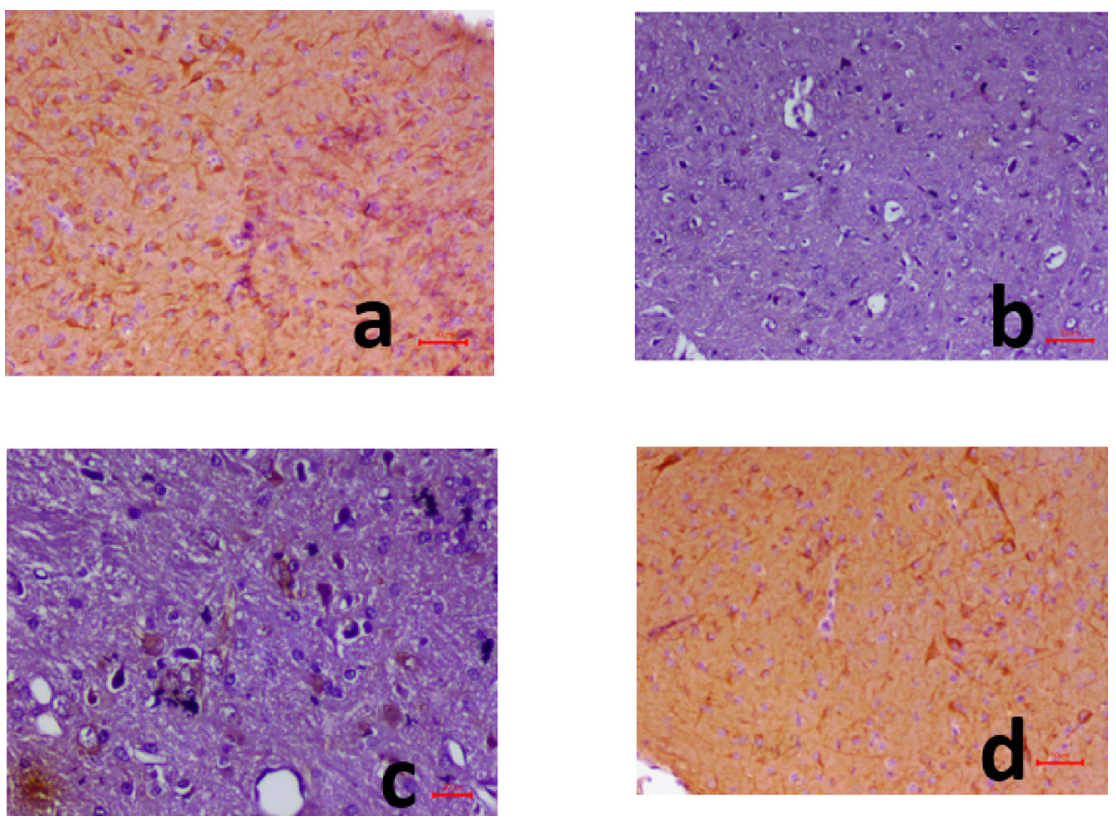

Fig. 8. Microscopic images from coronal sections of corpus striatum, showing TH immunoreactivity. (A) control, showed normal TH expression indicated in brown color (B) PD, revealed decreased TH expression signifying degeneration (C) PD+ CVS, showed moderate TH expression when compared to PD indicating significant restoration of degenerated neurons (D) CVS, observed to show normal TH expression (scale bar $20 \mu \mathrm{m})$

nigrostriatal pathway. However, after CVS administration for 30 days, significantly higher expression of $\mathrm{TH}$ was noticed when compared to the $\mathrm{PD}$ group. A remarkable restoration of degenerated neurons following CVS was noticed. The corpus striatum of CVS group appeared normal. The immunohistochemical investigation proved the efficacy of CVS in PD induced rats.

\section{DISCUSSION}

The present study was conducted to assess the protective role of CVS in rotenone-induced PD in rats with regard to behavioral variations, neurotransmitter alterations and histopathological changes. The authors used $3 \mathrm{mg} / \mathrm{kg}$ body weight of rotenone for 21 days to induce progressive neuronal loss in the corpus striatum, thereby producing nigro-striatal damage. Rotenone is a potent pesticide and its chronic administration (i.p.) has been widely used in experimental studies to induce pathophysiological features similar to that seen in PD patients $[6,20,21]$. Because of the lipophilic nature of rotenone, it can easily cross all biological membranes including the blood-brain barrier and can induce organ-specific damage. The rotenone model developed for PD induced nigro-striatal damage associated with Lewy body formation \& mitochondrial complex I inhibition, indicating its neurotoxic effect [22]. 
The behavioral alterations such as bradykinesia, postural instability, and rigidity revealed significant damage to the nigro-striatal neurons after rotenone administration [2]. The results of the present study showed that rotenone had a significant deteriorating effect on striatal neurons, thereby causing a decline in locomotion, general movement and motor coordination. The abovementioned results were consistent with the results from previous studies $[6,23,24]$. Interestingly, the authors found that CVS for 30 days improved the behavioral alterations in rotenoneinjected rats, providing significant protection for the neurons. Recently, in a case study repeated sessions of CVS were shown to relieve motor symptoms associated with PD with no serious adverse effect associated with CVS administration [13].

Rotenone is a well-known mitochondrial complex I inhibitor, and rotenone exposure causes rupture of the transport vesicles containing dopamine, which in turn leads to its release from dopaminergic neurons. Thus, rotenone produces dopamine dysfunction in the corpus striatum [15]. The dopamine dysfunction due to rotenone toxicity is important in producing motor deficits in PD. The findings of the present study showed significantly decreased dopamine neurotransmitter content in the rats with rotenone-induced PD when compared to control rats, indicating damage to the dopaminergic system. The reduced dopamine content was reversed by administration of CVS, thus preventing neuronal damage. A recent study in the 1-methyl-4phenyl-1,2,3,6-tetrahydropyridine-induced (MPTP) mice model of PD reported that dopamine neurotransmitter was elevated after CVS administration indicating the beneficial effect of CVS over the dopaminergic system [25]. Electrophysiological studies in rats have established that electrical stimulation of the vestibular apparatus modified the activity in the striatum [26, 27]. The current investigation on CVS showed positive effects on the brain function of rats with rotenone-induced PD. Nevertheless, it could be possible that CVS might bring some reorganizing effect on neurotransmission by enhancing dopamine release by striatal neurons, which in turn could interfere with the pathophysiological activity of neurons. It must be remembered that the important function of otoliths is perception of vertical gravitational information to the cortical areas [10]. To obtain accurate facts on how CVS offers benefits and the mechanisms involved, advanced studies in animals and humans are required.

The immunohistochemical analysis of brain sections revealed significant degeneration of the dopaminergic neurons in the nigro-striatal region following rotenone administration to rats. The histopathological findings of the study showed that rotenone had a significant degenerating effect on the striatal neurons, consistently with previous study results $[2,6,17,28]$. The TH neurons situated in the SN project their nerve terminals to the striatum. Consequently, the loss of dopaminergic neurons in the SN would result in the retraction of dopaminergic nerve terminals in the striatum region. The authors observed that rotenone administration significantly reduced the number of $\mathrm{TH}$-immunoreactive neurons in the dopamine (DA) nerve terminal of striatum, indicating neuronal loss in the SN. It is well-known that the loss of dopamine neurons in the $\mathrm{SN}$ and the decrease in dopamine nerve terminals in the striatum are considered to be the pathological hallmarks of PD. Interestingly, the authors found that CVS administration to rotenone-injected rats preserved the striatal dopaminergic nerve terminals from damage and rendered significant protection to dopaminergic neurons in the SN. However, the vestibulardeficient KCNE 1 mutant mouse was associated with overexpression of TH, suggesting a change in striatal functions by abnormalities in the vestibular apparatus [29]. The above-mentioned finding showed an interesting connection between the striatum and vestibular input. The striatum of basal ganglia is known to receive sensory information from the vestibular apparatus 
to initiate voluntary movements. Thus, in the present study, CVS might bring changes in striatal functions to help transmit the vestibular information to cortical and subcortical structures to achieve coordinated movements in PD-induced rats. In addition, CVS also possessed beneficial effects in relieving stress and depression, which are the common nonmotor symptoms associated with PD [11]. Based on the results of the present study and earlier reports, CVS appeared to be potently preventive against dopaminergic neurodegeneration in $\mathrm{PD}[10,12,25]$. The absence of any adverse effects with only CVS treatment on brain tissues further supported the relative safety of this effective intervention. The protective effects observed in this study could be extended further to promote the therapeutic application of CVS in protection against PD and environmental neurotoxicity caused by exposure to pesticides such as rotenone.

\section{CONCLUSION}

In summary, the present study demonstrated that CVS inhibited rotenone-induced dopaminergic neurodegeneration. The protective effects of CVS were most likely mediated through the enhancement of striatal dopamine release with neuropathological modifications. It can be concluded from the present study that CSV might be used as a neuroprotective measure in the prevention and treatment of motor deficits in PD. However, the identification of the exact molecular mechanism by which CSV enhanced the dopamine release would require further investigation.

Conflict of interest: The authors have specified that they have no conflicts of interest concerning the content of the present article.

\section{ACKNOWLEDGMENT}

The authors would like to thank Department of Research, SIMATS, India for providing technical support for the study.

\section{REFERENCES}

1. Bishnoi M, Chopra K, Kulkarni SK. Involvement of adenosinergic receptor system in an animal model of tardive dyskinesia and associated behavioural, biochemical and neurochemical changes. Eur J Pharmacol 2006; 552: 55-66.

2. Cannon JR, Tapias V, Na HM, Honick AS, Drolet RE, Greenamyre JT. A highly reproducible rotenone model of Parkinson's disease. Neurobiol Dis 2009; 34: 279-90.

3. Grimbergen YA, Munneke M, Bloem BR. Falls in Parkinson's disease. Curr Opin Neurol 2004; 17: 405-15.

4. Lerman SF, Bronner G, Cohen OS, Elincx-Benizri S, Strauss H, Yahalom G, et al. Catastrophizing mediates the relationship between non-motor symptoms and quality of life in Parkinson's disease. Disabil Health J 2019; 12: 673-8. 
5. Parkinson G. Disease survey steering committee. factors impacting on quality of life in Parkinson's disease: results from an international survey. Mov Disord 2002; 17: 60-7.

6. Betarbet R, Sherer TB, MacKenzie G, Garcia-Osuna M, Panov AV, Greenamyre JT. Chronic systemic pesticide exposure reproduces features of Parkinson's disease. Nature Neurosci 2000; 3: 1301-6.

7. Smith TS, Parker JW, Bennett JJ. L-dopa increases nigral production of hydroxyl radicals in vivo: potential Ldopa toxicity? Neuroreport 1994; 5: 1009-11.

8. deSouza RM, Moro E, Lang AE, Schapira AH. Timing of deep brain stimulation in Parkinson disease: a need for reappraisal? Annal Neurol 2013; 73: 565-75.

9. Utz KS, Dimova V, Oppenländer K, Kerkhoff G. Electrified minds: transcranial direct current stimulation (tDCS) and galvanic vestibular stimulation (GVS) as methods of non-invasive brain stimulation in neuropsychology — a review of current data and future implications. Neuropsychologia 2010; 48: 2789-810.

10. Smith PF. Vestibular functions and Parkinson's disease. Front Neurol 2018; 9: 1085.

11. Sailesh SK, Archana R, Mukkadan JK. Controlled vestibular stimulation: a physiological method of stress relief. J Clin Diagn Res 2014; 8: BM01-02.

12. Wilkinson D, Podlewska A, Banducci SE, Pellat-Higgins T, Slade M, Bodani M, et al. Caloric vestibular stimulation for the management of motor and non-motor symptoms in Parkinson's disease: intention-totreat data. Data Brief 2019; 25: 104228.

13. Wilkinson D, Podlewska A, Banducci SE, Pellat-Higgins T, Slade M, Bodani M, et al. Caloric vestibular stimulation for the management of motor and non-motor symptoms in Parkinson's disease. Parkinsonism Relat Disord 2019; 65: 261-6.

14. Nishiike S, Takeda N, Kubo T, Nakamura S. Neurons in rostral ventrolateral medulla mediate vestibular inhibition of locus coeruleus in rats. Neuroscience 1997; 77: 219-32.

15. Nishiike S, Takeda N, Uno A, Kubo T, Yamatodani A, Nakamura S. Cholinergic influence on vestibular stimulation-induced locus coeruleus inhibition in rats. Acta oto-laryngologica 2000; 120: 404-9.

16. Ludolph A, He F, Spencer P, Hammerstad J, Sabri M. 3-Nitropropionic acid-exogenous animal neurotoxin and possible human striatal toxin. Can J Neurol Sci 1991; 18: 492-8.

17. Kishore Kumar SN, Deepthy J, Saraswathi U, Thangarajeswari M, Yogesh Kanna S, et al. Morinda citrifolia mitigates rotenone-induced striatal neuronal loss in male Sprague-Dawley rats by preventing mitochondrial pathway of intrinsic apoptosis. Redox Report 2017; 22: 418-29.

18. Gu M-J, Jeon J-H, Oh MS, Hong S-P. Measuring levels of biogenic amines and their metabolites in rat brain tissue using high-performance liquid chromatography with photodiode array detection. Archives Pharmacal Res 2016; 39: 59-65.

19. Pangestiningsih TW, Wendo WD, Selan YN, Amalo FA, Ndaong NA, Lenda V. Histological features of catecholaminergic neuron in substantia nigra induced by paraquat dichloride (1,1-dimethyl-4,4 bipyridinium) in Wistar rat as a model of Parkinson disease. Indonesian J Biotechnol 2014; 19: 91-8.

20. Johnson ME, Bobrovskaya L. An update on the rotenone models of Parkinson's disease: their ability to reproduce the features of clinical disease and model gene-environment interactions. Neurotoxicology 2015; 46: 101-16.

21. Ren Y, Liu W, Jiang H, Jiang Q, Feng J. Selective vulnerability of dopaminergic neurons to microtubule depolymerization. J Biol Chem 2005; 280: 34105-12.

22. Sanders LH, Greenamyre JT. Oxidative damage to macromolecules in human Parkinson disease and the rotenone model. Free Radical Biol Med 2013; 62: 111-20.

23. Nehru B, Verma R, Khanna P, Sharma SK. Behavioral alterations in rotenone model of Parkinson's disease: attenuation by co-treatment of centrophenoxine. Brain Res 2008; 1201: 122-7. 
24. von Wrangel C, Schwabe K, John N, Krauss JK, Alam M. The rotenone-induced rat model of Parkinson's disease: behavioral and electrophysiological findings. Behav Brain Res 2015; 279: 52-61.

25. Sailesh KS, Archana R. Effect of caloric vestibular stimulation on brain neurotransmitters in an MPTPinduced mouse model of Parkinson's disease. Int J Res Pharm Sci 2019; 10: 40-4.

26. Rancz EA, Moya J, Drawitsch F, Brichta AM, Canals S, Margrie TW. Widespread vestibular activation of the rodent cortex. J Neurosci 2015; 35: 5926-34.

27. Stiles L, Reynolds JN, Napper R, Zheng Y, Smith PF. Single neuron activity and c-Fos expression in the rat striatum following electrical stimulation of the peripheral vestibular system. Physiological Report 2018; 6: e13791.

28. Alam M, Schmidt W. Rotenone destroys dopaminergic neurons and induces parkinsonian symptoms in rats. Behav Brain Res 2002; 136: 317-24.

29. Richter A, Ebert U, Nobrega J, Vallbacka J, Fedrowitz M, Löscher W. Immunohistochemical and neurochemical studies on nigral and striatal functions in the circling (ci) rat, a genetic animal model with spontaneous rotational behavior. Neuroscience 1999; 89: 461-71. 